\title{
Zinc Nanoparticles-equipped Bioelectronic Nose Using a Microelectrode Array for Odorant Detection
}

\author{
Qian Zhang, ${ }^{*}, * *$ Diming Zhang, ${ }^{*}, * *$ Nantao LI,,$* *$ Yanli Lu, $* * *$ Yao YAo, $*, * *$ Shuang LI,,$* *$ \\ and Qingjun LiU,***
}

*Biosensor National Special Laboratory, Department of Biomedical Engineering, Zhejiang University, Hangzhou, 310027, P. R. China

**Cyber Innovation Joint Research Center, Zhejiang University, Hangzhou, 310027, P. R. China

\begin{abstract}
Bioelectronic noses, such as olfactory cell- and receptor-based biosensors, have important applications for biomimetic odorant detection in various fields. Here, a nanoparticle-equipped biosensor was designed to record extracellular potentials from olfactory receptor cells effectively. In this research, a microelectrode array (MEA) was combined with olfactory epitheliums as the olfactory biosensor to record electrophysiological signals of receptor cells in the epitheliums. Zinc nanoparticles (NanoZn) were employed along with the biosensor for different kinds of odorant measurements, which improved the electrophysiological responses to odor molecules. The NanoZn-equipped biosensor showed greater performance, such as a higher sensitivity and a larger signal-to-noise ratio, than that without the nanoparticles. Thus, this approach provided a promising method to improve the detecting performance of biosensors based on olfactory cells and receptors, which would bring broad application prospects for bioelectronic noses in environmental monitoring, food analysis, and healthcare diagnosis.
\end{abstract}

Keywords Bioelectronic nose, zinc nanoparticle, microelectrode array, signal-to-noise ratio

(Received August 28, 2015; Accepted October 29, 2015; Published April 10, 2016)

\section{Introduction}

Bioelectronic noses are artificial olfactory sensing devices for odorant detections and recognitions. They can percept odor molecules both qualitatively and quantitatively, by combining olfactory elements, such as receptors, cells, and tissues, to physical or chemical sensors. ${ }^{1-3}$ With well-persevered biological selectivity and sensitivity, bioelectronic devices have great potentials in fields of medicine, environment, food industry, and the military. ${ }^{4-6}$ Among these bio-devices, olfactory cell-based biosensors, which combine olfactory cells and even olfactory tissues with sensor chips, are widely studied as a kind of typical bioelectronic noses. ${ }^{7-9}$ These biosensors can well-mimic the biological perception of olfactory receptors expressed on cells. Both natural expressed and bioengineering expressed olfactory receptors can bind with odor molecules and transform the biochemical binding into electrophysiological signals, which can then be detected by physicochemical sensors, such as, microelectrode arrays and field-effect transistors. ${ }^{10-12}$

However, the detecting capabilities of these bioelectronic noses were often influenced by varieties of inevitable noises, such as intrinsic noises of devices, random noises, biochemical shot noises, and coupling noises between biological elements and electronic devices. ${ }^{13,14}$ These noises could overwhelm small response signals, reduce the signal-to-noise ratio (SNR), and

† To whom correspondence should be addressed.

E-mail: qjliu@zju.edu.cn then weaken the detection sensitivity, which all greatly limited applications of these biosensors. ${ }^{15}$ Thus, many efforts were made to reduce noises and to amplify signals so as to improve the sensitivity of bioelectronics noses. For instance, nanostructures, such as nanowires and nanoparticles, were often fabricated on electrode surfaces to increase compact attachment and to minimize any extra influence from the bath solution. ${ }^{16,17}$ But, most methods reported seemed to be complicated and expensive to mass produce, thus limiting their applications.

Olfactory receptors were reported to function as metalloproteins for their metal binding sites. ${ }^{18}$ Metals are likely to play important roles in both odorant detection and recognition. As one of the most studied metals associated with olfactory receptors, zinc is an essential metal element for organisms, and has been found in olfactory bulbs and olfactory epithelium. ${ }^{19,20}$ Clinical studies even suggested that dietary zinc deficiency could cause diseases, such as anosmia, which indicated the importance of zinc in the olfactory system. Recently, animal studies reported that colloid zinc nanoparticles (NanoZn) isolated from blood could enhance the response signals of olfactory receptor cells. ${ }^{21}$ Based on these results, it could be inferred that nanoparticles, especially NanoZn, might play a role in improving the sensitivity of bioelectronics noses.

With natural receptors well preserved and basic structures of neuronal population kept integrated, the electrophysiological activities of receptor cells could be recorded by olfactory epithelium combined with microelectrode arrays (MEAs) in our previous study. ${ }^{8,22}$ MEA could be employed to better capture the dynamics of native biological elements, and to provide a 


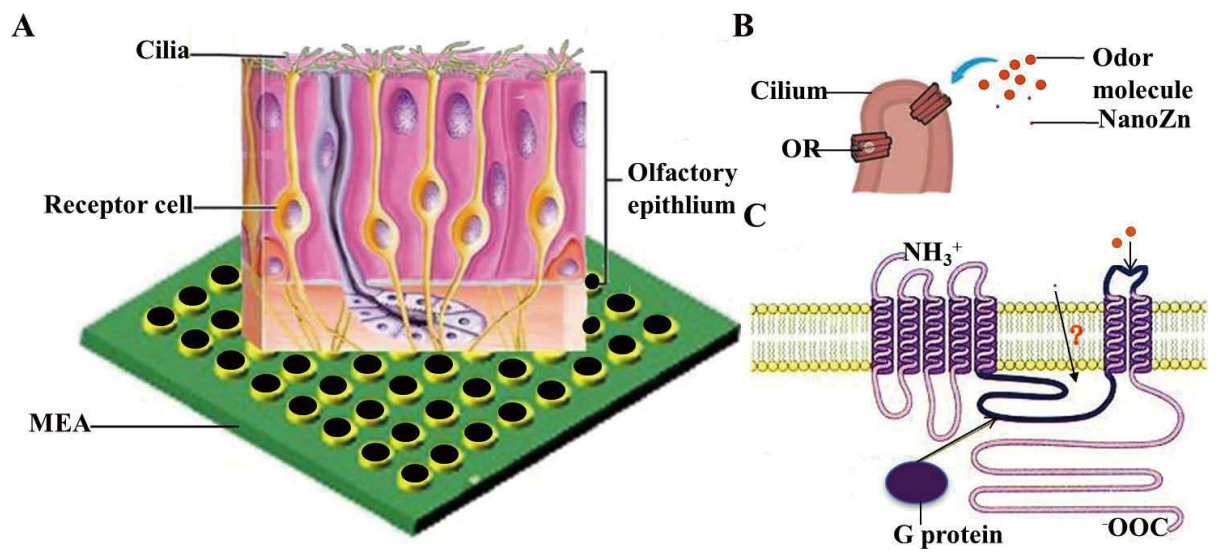

Fig. 1 Schematic of the olfactory cell-based biosensor and its primary olfactory perception mechanism with NanoZn. (A) Sensing portion of the biosensor. (B) Schematic of one cilium on the olfactory epithelium. (C) Structure of the olfactory receptor on the cytomembrane and its odor perception mechanism.

means to detect non-destructively, record from many individual cells or synapses simultaneously, and to study spatio-temporal patterns of electrical activity in dissociated cells or tissues. For biosensor applications, MEA coupled with biological elements in vitro could be considered as a more advanced and high sensitive system, which strongly depends on the compatibility between its electrodes and the bio-recognition elements. ${ }^{23-25}$ As a result, it was a valuable tool for long-time network-level electrophysiological investigations, but also persecuted by noises. Here, a NanoZn-equipped biosensor, based on olfactory receptor cells combined with MEA, was further developed in order to explore analytical methods for improving the performance of olfactory biosensors. In experiments, the effect of NanoZn with the biosensor was investigated at different concentrations, and then the optimized concentration of the nanoparticles could be found for different kinds of odorant measurements. This study mainly focused on exploring whether the enhancement of NanoZn can be used in the biosensor design, and which point of the enhancement can be used to improve the performance of the biosensor. Results suggested that the equipment of the NanoZn could improve the performance of the bioelectronic nose, such as SNR and sensitivity, by the effect on the olfactory biosensing process, itself, which provided a more sensitive platform for odorant detections, and might stimulate great progress for applications of bioelectronic noses.

\section{Experimental}

Preparation of the olfactory cell-based biosensor

Sprague-Dawley rats with weights of $250-300 \mathrm{~g}$ were purchased from the Laboratory of Animal Research Center (Zhejiang Province, China). After being anaesthetized, the olfactory mucosa of the rat was removed, as described in our previous work. $^{22}$ The isolated epithelium (about $5 \times 5 \mathrm{~mm}$ ) was rinsed with Ringer's solution and placed with the ciliated side up on the microelectrode area of MEA, as shown in Fig. 1. The MEA used in this study was composed of 64 microelectrodes, which were coated with platinum black so as to reduce noises of the electrodes. The electrodes could couple with olfactory receptor cells in the epithelium and receive responses of them to odor stimuli, thus realizing the real-time recording of odorantinduced signals at different sites of the epithelium. After

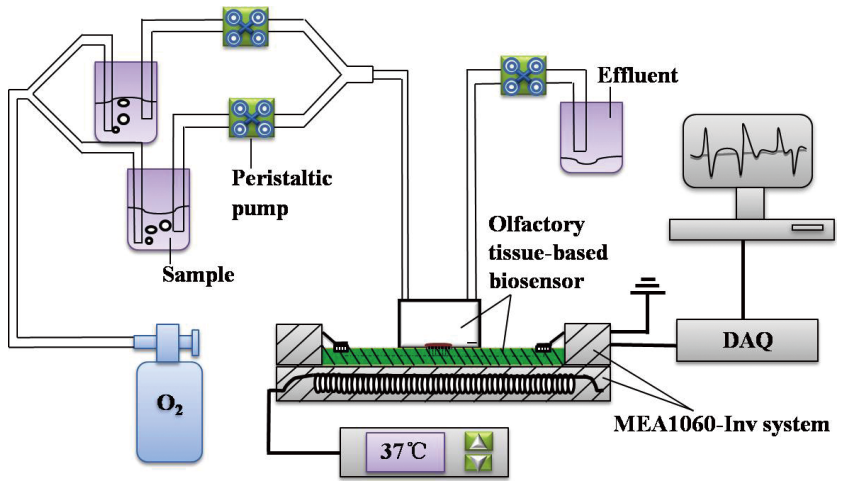

Fig. 2 Schematic of the detection platform of the olfactory biosensor.

rinsing, the buffer solution was removed from the MEA chamber and the epithelium could be fixed by a plastic ring-shaped frame covered with a tightly stretched piece of mesh.

\section{Olfactory cell-based biosensor detection platform}

The whole olfactory detecting system is shown in Fig. 2. The fixed epithelium was perfused with oxygenated Ringer's solution at a flow rate of $10 \mu \mathrm{L} / \mathrm{min}$ by peristaltic pumps (FPU500, OMEGA, USA). The odor compounds and NanoZn were bath-applied through an active perfusion system. The responses of the odor compounds were recorded using the MEA1060-Inv system from Multichannel Systems (MCS, Reutlingen, Germany). The analog extracellular neuronal signals were AC amplified, then sampled at $20 \mathrm{kHz}$, and stored on a compatible computer for subsequent off-line analysis. All recordings were subsequently subjected to analysis using MATLAB (MathWorks, Natick, MA).

\section{Chemicals and conditions for odorant detection}

The obtained responses were elicited by typical odor stimuli with the standard phosphate buffer solution (PBS) and NanoZn. Different concentrations of isoamyl acetate and acetic acid (Sigma, St. Louis, MO) were chosen as odor stimuli to olfactory cells. The concentrations of isoamyl acetate and acetic acid were $10^{-7}, 10^{-5}$ and $10^{-3} \mathrm{M}$. NanoZn was obtained from Purest Colloids Inc. (United States), which was filtered through 


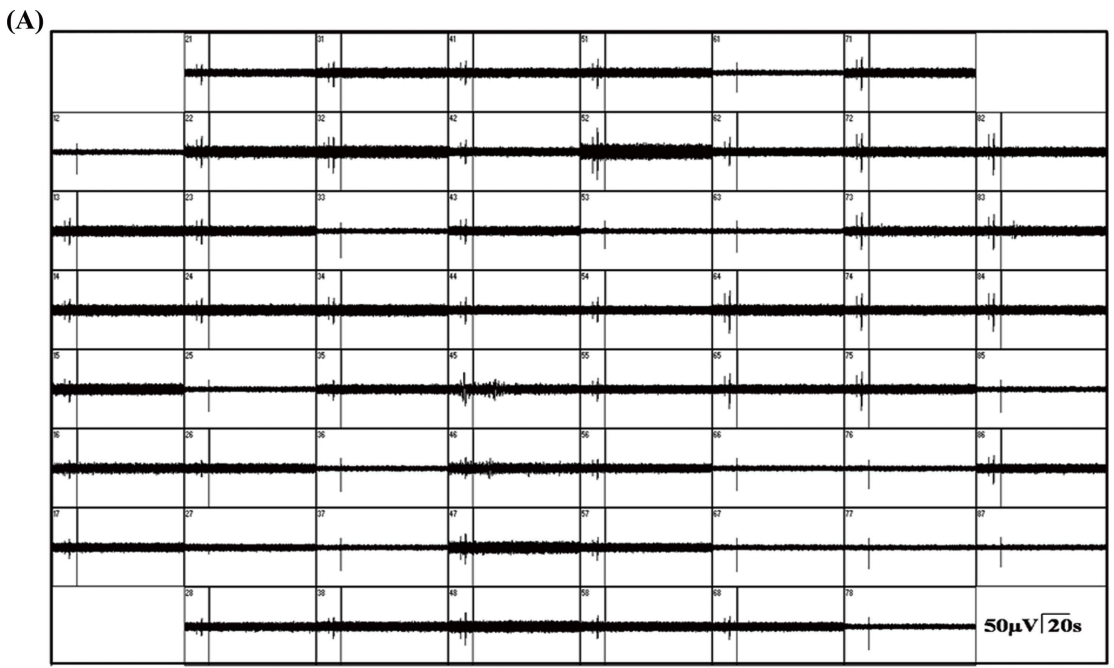

(B)
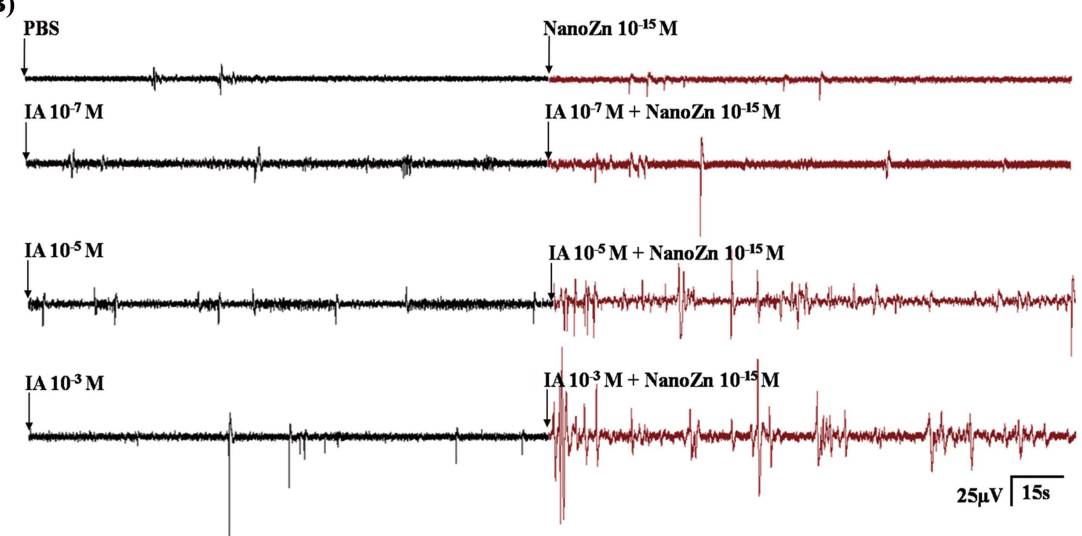

Fig. 3 Detection results of the olfactory cell-based biosensor to isoamyl acetate stimuli at different concentrations. (A) Example of 60-channel signal responses of the biosensor under stimulations of isoamyl acetate by $10^{-7} \mathrm{M}$ mixed with NanoZn by $10^{-10} \mathrm{M}$. (B) Single-channel signals under stimulations of isoamyl acetate. Signals in red showed response signals detected by a biosensor equipped with NanoZn, while the black ones represented that without NanoZn as a control.

$0.22-\mu \mathrm{m}$ Millipore Express Filters and centrifuged at $40000 \mathrm{~g}$ for $90 \mathrm{~min}$ at $18^{\circ} \mathrm{C},{ }^{21}$ then the supernatant fluid was used for following biosensor experiments. The concentrations of NanoZn used in this research were $10^{-15}, 10^{-14}, 10^{-12}, 10^{-10}, 10^{-8}$, $10^{-7}, 10^{-6}$ and $10^{-5} \mathrm{M}$. PBS was purchased from Sigma, and all solvents used for dilution were deionized water. Native and NanoZn-induced electrophysiological activities of olfactory epithelium were recorded for $150 \mathrm{~s}$ as the control. After odorants were perfused into the testing chamber, the recording also lasted for about $150 \mathrm{~s}$. Then, stimuli were thoroughly washed out from the chamber by PBS. All solutions were added by peristaltic pumps, and selective valves were controlled by a personal computer. All recordings were performed at $25^{\circ} \mathrm{C}$ (room temperature).

\section{Results}

Detecting effect of the nanoparticle-equipped biosensor

By combining the olfactory epithelium with the MEA, a bioelectronic nose was established to measure real-time extracellular potentials caused by interactions between odor molecules and odor receptors on different sites of the epithelium (Fig. 3A). It could be seen that the electrophysiological activities of receptor cells in the intact epithelium could be effectively analyzed by the multi-channel recording. In experiments, the effects of NanoZn equipped with the biosensor were first examined under stimulations of isoamyl acetate, which was a covalent compound and a representative of aromatic substances, and had been broadly utilized in olfactory researches. ${ }^{26}$ Through comparisons of the two-dimensional results in the presence of nanoZn with that in the absence of the particles (Fig. S1, Supporting Information), it could be found that there were many channels appeared responses and larger signals in the channel with a higher firing rate after the addition of nanoZn. As a result, one of multiple channels with the highest firing rate in each recording was chosen for further analyzing and processing, and it could be seen that, under the treatment of NanoZn at $10^{-15} \mathrm{M}$, the biosensor could be more activated by isoamyl acetate, as shown in Fig. 3B.

To quantitatively analyze the effect of NanoZn on the responses of the biosensor to isoamyl acetate, we subsequently identified and extracted effective extracellular signals of the preprocessed single-channel signals. Figure 4 is a statistical comparison of the peak-to-peak amplitudes and inter-stimulus intervals (ISIs) of effective signals obtained, respectively. Consistent with Fig. 3B, the amplitudes of extracellular signals intensified with increasing isoamyl acetate concentrations 

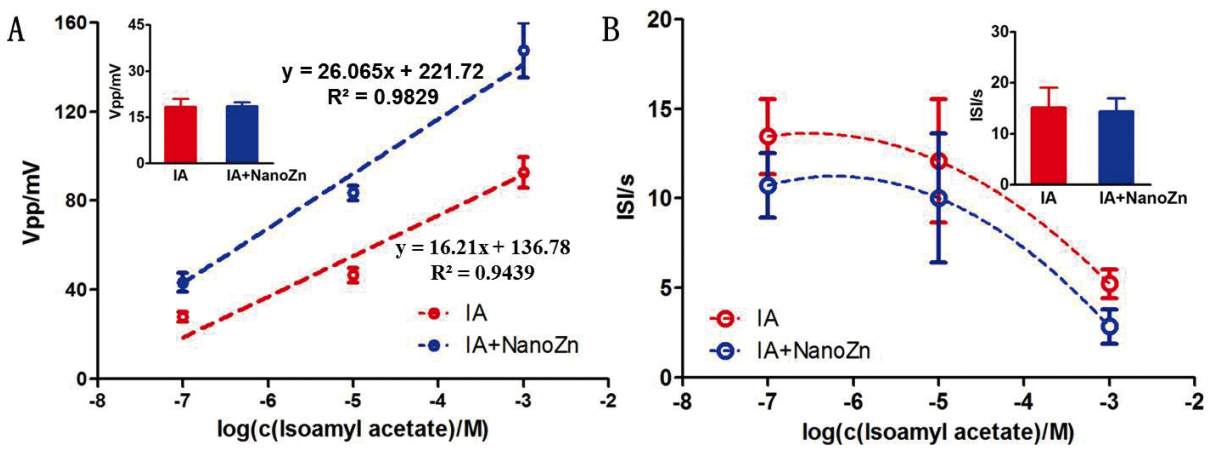

Fig. 4 Statistical comparison of the peak-to-peak amplitudes (A) and ISIs (B) under stimulations of different concentrations of isoamyl acetate (IA) with and without $10^{-15} \mathrm{M}$ NanoZn equipment (mean $\pm \mathrm{SD}$ ).
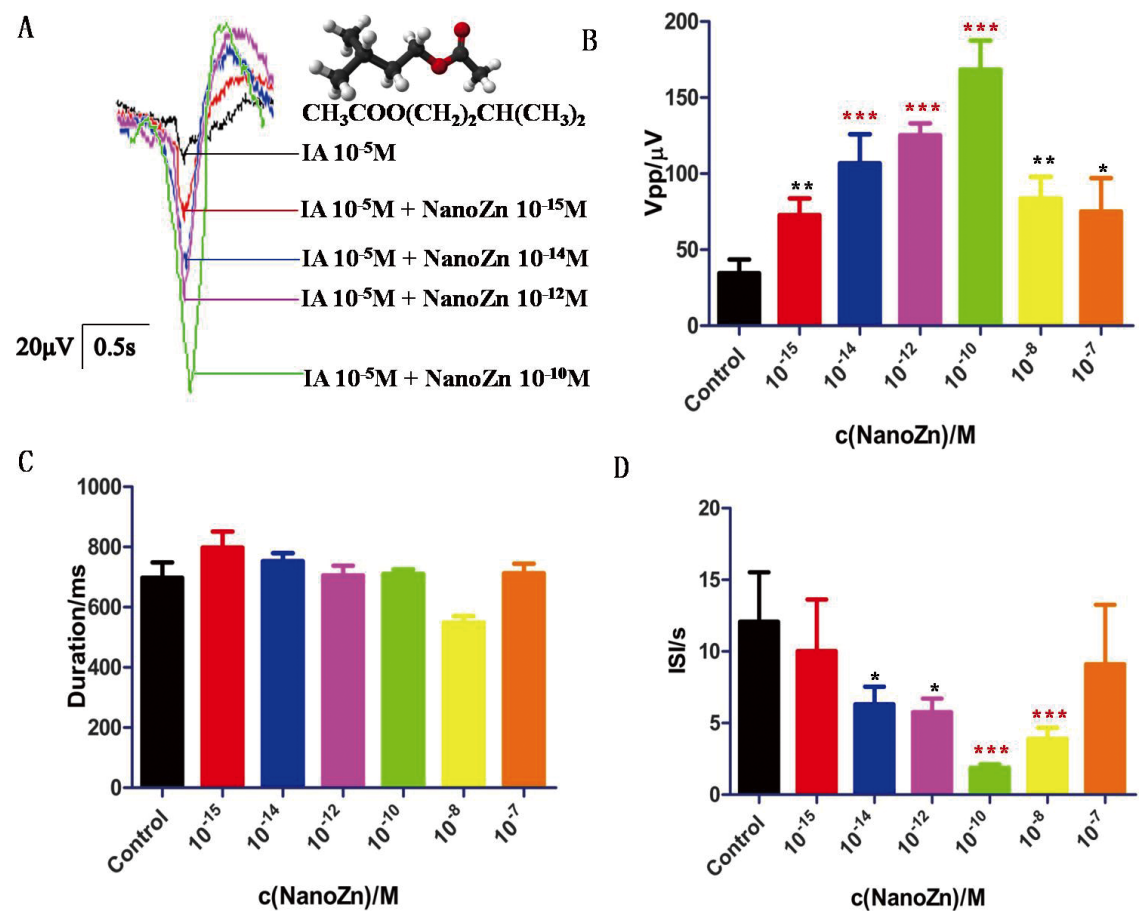

D

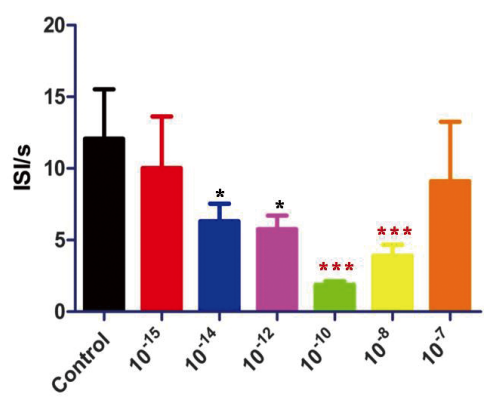

Fig. 5 Effective signal responses of the biosensor equipped with different concentrations of NanoZn to $10^{-5} \mathrm{M}$ isoamyl acetate. (A) Average characteristic signals obtained by clustering effective response signals. The insert figure was the structure of isoamyl acetate. (B, C and D) Statistical comparison of peak-to-peak amplitudes $\left(V_{\mathrm{pp}}\right)$, durations and ISIs. (mean $\pm \mathrm{SD}$ ) The control trail represented response values detected by non-equipped olfactory cell-based biosensor.

(Fig. 4A), while ISIs decreased (Fig. 4B). On the other side, without isoamyl acetate stimuli, both amplitudes and ISIs recorded by the biosensor equipped with NanoZn were almost the same as that without NanoZn, as shown in histogram insets. From Fig. 3B, it could be found that the noise levels were similar for all stimulations. Therefore, the larger were the signals, the greater was the SNR. Taking $S / N \geq 3$ as the condition of signal extraction, it could be calculated from statistical results that the sensitivity of the biosensor was increased by greater than 1.6 times. As a result, the presence of NanoZn gave rise to an enhancement of SNR and the sensitivity of the biosensor, which indicated more effective detection capacity of the biosensor equipped with NanoZn.
Concentration optimization of nanoparticles with the biosensor for covalent odorant detection

To further explore the enhanced effect of NanoZn on odorant detection of the olfactory biosensor, the influences of NanoZn at different concentrations were also investigated. Taking responses to isoamyl acetate alone as the control, the effects of NanoZn at different concentrations were detected and analyzed with fixing isoamyl acetate at $10^{-5} \mathrm{M}$. After extracting $(S / N \geq 3)$ and clustering effective response signals by a K-mean clustering algorithm, the average characteristic signals obtained were found, as displayed in Fig. 5A, showing that increasing the concentration of NanoZn could lead to stronger characteristic signals in a certain range.

As basic characteristics, the waveform, amplitude, duration and the firing rate (or ISI) of extracellular potentials were usually used to represent the response intensity to stimuli. ${ }^{27}$ 


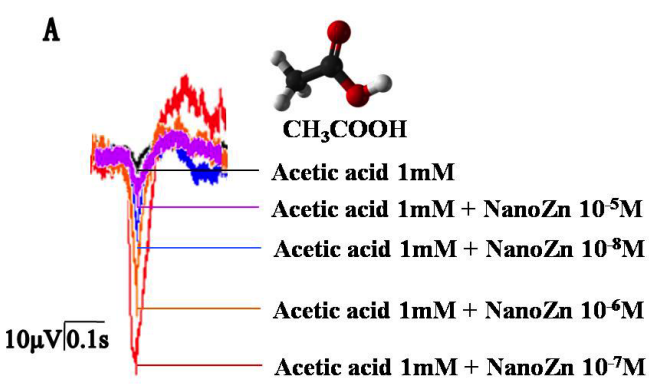

C

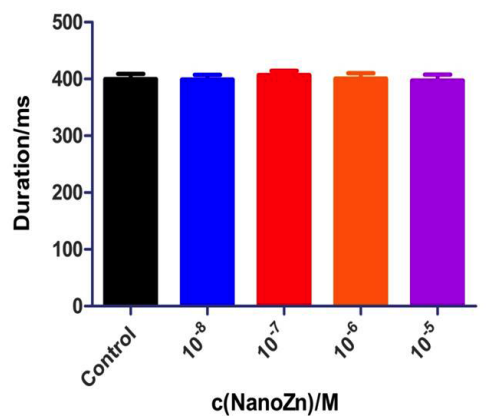

B

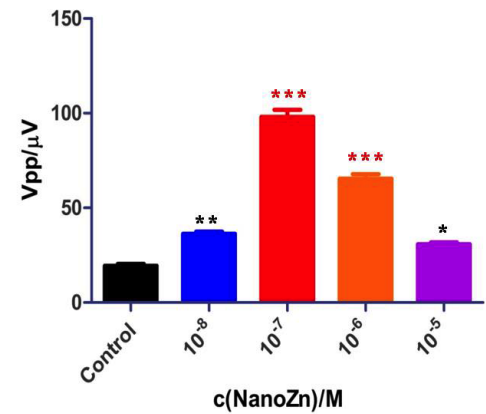

D

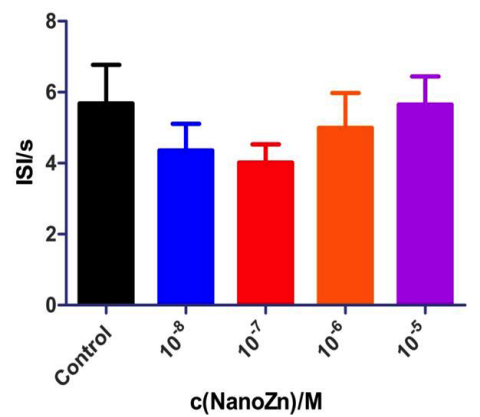

Fig. 6 Effective signal responses of the biosensor equipped with different concentrations of NanoZn to $10^{-3} \mathrm{M}$ acetic acid. (A) Average characteristic signals obtained by clustering effective response signals. The insert figure shows the structure of acetic acid. (B, C and D) Statistical comparison of peak-to-peak amplitudes $\left(V_{\mathrm{pp}}\right)$, durations and ISIs. (mean $\pm \mathrm{SD}$ ) The control trail represented response values detected by non-equipped olfactory cell-based biosensor.

In this study, the peak-to-peak amplitudes, durations and ISIs of effective signals under stimulations were also analyzed. It can be seen quantitatively from Figs. 5B and 5C that the amplitudes of isoamyl acetate were significantly enhanced by NanoZn in a certain range of concentrations, while the durations fluctuated slightly. Besides, Fig. 5D reflected that ISIs diminished with increasing concentrations of NanoZn in a certain range, which meant that the firing frequency of the biosensor's responses increased as well. On the other hand, when the concentration of NanoZn increased beyond $10^{-8} \mathrm{M}$, the enhanced effect of NanoZn gradually decreased, which indicated the limits of NanoZn's enhanced effect. In summary, increasing the concentration of NanoZn in a certain range could enhance the responses of the biosensor in the amplitudes and firing frequency. Specifically, the average peak-to-peak amplitudes of the responses were increased by nearly four times compared to the control when NanoZn was at $10^{-10} \mathrm{M}$, indicating an optimal concentration of NanoZn equipped with the biosensor for detection of this covalent odorant.

\section{Concentration optimization of nanoparticles with the biosensor} for ionic odorant detection

To explore whether the enhancement effect and the optimal dose of NanoZn with the biosensor was universal or not, concentration optimization experiments were also carried out under stimulations of acetic acid, which is an ionic compound and also a typical kind of odor stimuli that serve as a representative in odorant detection. ${ }^{28}$ Figure $6 \mathrm{~A}$ shows the characteristic signals of electrophysiological responses recorded by the biosensor to acetic acid with NanoZn. The responses were also increased by NanoZn at different concentrations, and the average SNR obtained from response signals could be approximately increased by 5 times when equipped with $10^{-7} \mathrm{M}$ NanoZn. As shown in Figs. 5B, 5C and 5D, NanoZn showed a significant enhancement in amplitude of the responses from biosensor as well, which was similar to its enhancement for isoamyl acetate detection. However, when the concentration of NanoZn increased beyond $10^{-7} \mathrm{M}$, the enhancement of the nanoparticles gradually changed from increasing into decreasing, showing that the optimal concentration of NanoZn was around $10^{-7} \mathrm{M}$ in the enhancement for acetic acid detection, which was different from that for isoamyl acetate detection. As a result, the enhancement effect of the nanoparticles equipped with the biosensor seemed to be universal for odorant detection, indicating broad-spectrum characteristics in improvement for the performance of the olfactory biosensor. On the other hand, the optimal concentration of NanoZn varied with different odorants, which might also be helpful for odorant discrimination with the nanoparticles-equipped biosensor. Thus, NanoZn could be applied in plenty of odorant detections by the olfactory cellbased biosensor.

\section{Discussion}

Over the last couple of decades, significant efforts have been made to develop bioelectronic noses that combine biosensing components to physicochemical transducers, in the field of environmental monitoring, food safety and medical diagnosis. ${ }^{6,25,29-31}$ Although bioelectronic noses have good selectivity and sensitivity, they still suffer from intrinsic and external noise interference. Thus, various ultrasensitive transducers (e.g., field-effect transistor, surface plasmon resonance and quartz crystal microbalance) have been used to detect signals from biomolecular binding. However, it has been known that the detection limit of bioelectronic noses depends not only on measurement technique and detecting instruments, but also on signals obtained from biological elements. ${ }^{32-34}$ Thus, 
it can be assumed that enhancing original biological signals will improve the odorant detection of bioelectronic noses, especially olfactory cell-based biosensors. In our study, we showed the design of a nanoparticle-equipped biosensor for odorant detection. The electrophysiological enhancement of NanoZn was utilized in the biosensor design for some performance improvements. In this study, the physiological signals of olfactory tissues with an enhancement of nanoZn were used as the responses of the biosensor for odorant detections. The signal features, such as the amplitude, duration, and firing rate, were investigated and chosen as the responses of the improved biosensor.

Studies have found that many kinds of metals, such as $\mathrm{Cu}, \mathrm{Mn}$ and $\mathrm{Fe}$, besides $\mathrm{Zn}$, may also have effects on olfaction for their relations with neural systems. ${ }^{9,35,36}$ The effects were related to the states, valences and sizes of metals. For example, copper ions could react with specific odorants to enhance the responses of MOR244-3, ${ }^{37}$ while copper nanoparticles could not improve the olfactory sensing. ${ }^{21}$ In this study, the improvement of odorant detection sensitivity of the olfactory cell-based biosensor was realized by combining NanoZn with olfactory cells. Our results showed that both the average amplitude and the firing frequency of the responses to odor stimuli were increased when the olfactory cell-based biosensor was equipped with suitable concentrations of NanoZn. In a certain range, the larger was the concentration of the odorant, the stronger was the effect. Besides, this NanoZn equipped olfactory biosensor had shown dose-dependence and broad-spectrum characteristics in measurements, indicating wide applications in the field of olfactory biosensors.

According to studies about the mechanism of the enhanced effect of NanoZn, it has been speculated that NanoZn acted as a "protein metal" when NanoZn was combined with olfactory receptor cells. One single receptor/Golf (olfactory-related G protein) complex may be composed of two receptors, one zinc particle and one $\mathrm{G}_{\text {olf. }}{ }^{38}$ Responses under odor stimulations mixed with NanoZn came from two olfactory receptors, and transmitted by one Golf, making signals easier to superimpose. As a result, the amplitude and the firing frequency of response signals could both be increased, while the waveform of the signals fluctuated slightly. Besides, the enhancement did not exist when NanoZn was added alone without odor molecules (Fig. 3B), because the receptor/ $\mathrm{G}_{\text {olf }}$ complexes were not elicited. Moreover, when under stimulations of different kinds of odorants, the optimal concentrations of NanoZn for detection was different, which might be due to different amounts of Golf mobilized by combination of olfactory receptors with covalent and ionic odor molecules. This speculation indicated that our method could also be used for other olfactory cell- and receptor-based biosensors to improve the odorant detection of bioelectronic noses. As a result, this research provided an experiment basis for olfactory perception mechanisms of metal nanoparticles. The approach could be applied to more explorations with different kinds and sizes of metals to further expand the application fields of bioelectronic noses.

\section{Conclusion}

In this research, an olfactory cell-based biosensor was equipped with different concentrations of NanoZn so as to improve its odorant detecting performance. The physiological signals of olfactory tissues with enhancement of nanoZn were used as the responses of the biosensor for odorant detections. The results showed that the responses of the biosensor, especially amplitudes of electrophysiological signals, could be increased significantly by the nanoparticles in odorant detections for both isoamyl acetate and acetic acid, indicating broad-spectrum characteristics in improvements for the performance of olfactory biosensors. The NanoZn-equipped biosensor was demonstrated with a higher SNR and a lower detection limit than that without the nanoparticles, providing a well-performed sensing platform to further fabricate bioelectronic noses. However, the optimal concentration of NanoZn varied with different odorants, which might also be helpful for odorant discrimination with the biosensor. In summary, this study offers a potential method to investigate olfactory perception mechanisms and interactions between nanoparticles and olfactory receptor cells.

\section{Acknowledgements}

This work was supported by the National Natural Science Foundation of China (Grant No. 81371643), the Zhejiang Provincial Natural Science Foundation of China for Distinguished Young Scholars (Grant No. LR13H180002).

\section{Supporting Information}

Supplementary figure to compare the multi-channel responses to isoamyl acetate in the presence of nanoZn with that in the absence of the particles (Fig. S1). As shown in the figure, Fig. S1A represented multi-channel responses of the biosensor in 2 min without nanoZn while Fig. S1B displayed that with nanoZn. This material is available free of charge on the Web at http://www.jsac.or.jp/analsci/.

\section{References}

1. Q. Liu, H. Cai, Y. Xu, Y. Li, R. Li, and P. Wang, Biosens. Bioelectron., 2006, 22, 318.

2. B. Raman, J. L. Hertz, K. D. Benkstein, and S. Semancik, Anal. Chem., 2008, 80, 8364.

3. S. H. Lee and T. H. Park, Biotechnol. Bioprocess Eng., 2010, 15, 22.

4. P. Wang, Q. Liu, Y. Xu, H. Cai, and Y. Li, Sens. Actuators, A, 2007, 139, 131.

5. Q. Liu and P. Wang, "Cell-based Biosensors: Principles and Applications", 2009, Artech House.

6. E. H. Oh, H. S. Song, and T. H. Park, Enzyme Microb. Technol., 2011, 48, 427.

7. S. H. Lee, S. B. Jun, H. J. Ko, S. J. Kim, and T. H. Park, Biosens. Bioelectron., 2009, 24, 2659.

8. Q. Liu, N. Hu, W. Ye, H. Cai, F. Zhang, and P. Wang, Biosens. Bioelectron., 2011, 27, 12.

9. O. Civelli, R. K. Reinscheid, Y. Zhang, Z. Wang, R. Fredriksson, and H. B. Schiöth, Annu. Rev. Pharmacol. Toxicol., 2013, 53, 127.

10. R. Glatz and K. Bailey-Hill, Prog. Neurobiol., 2011, 93, 270.

11. C. Zhang, J. Yan, Y. Chen, C. Chen, K. Zhang, and X. Huang, Biotechnol. Adv., 2014, 32, 290.

12. C. Wehrenfennig, M. Schott, T. Gasch, R. A. Düring, A. Vilcinskas and C.-D. Kohl, Anal. Bioanal. Chem., 2013, 405, 6389.

13. A. Hassibi, H. Vikalo, and A. Hajimiri, J. Appl. Phys., 2007, 102, 014909.

14. A. D. Wilson, Sensors, 2013, 13, 2295. 
15. A. Poghossian, S. Ingebrandt, A. Offenhäusser, and M. Schöning, Semin. Cell Dev. Biol., 2009, 20, 41.

16. A. T. Sage, J. D. Besant, B. Lam, E. H. Sargent, and S. O. Kelley, Acc. Chem. Res., 2014, 47, 2417.

17. P. Nathalie, J. Biochips Tissue Chips, 2011, S1, 001.

18. J. Wang, Z. A. Luthey-Schulten, and K. S. Suslick, Proc. Natl. Acad. Sci., U. S. A., 2003, 100, 3035.

19. C. Frederickson, L. Giblin, A. Krezel, D. McAdoo, R. Mueller, Y. Zeng, R. Balaji, R. Masalha, R. Thompson, and C. Fierke, Exp. Neurol., 2006, 198, 285.

20. W. Maret, Adv. Nutr., 2013, 4, 82.

21. N. Viswaprakash, J. C. Dennis, L. Globa, O. Pustovyy, E. M. Josephson, P. Kanju, E. E. Morrison, and V. J. Vodyanoy, Chem. Senses, 2009, 34, 547.

22. Q. Liu, F. Zhang, N. Hu, H. Wang, K. J. Hsia, and P. Wang, J. Bionic Eng., 2012, 9, 494.

23. G. W. Gross, B. K. Rhoades, H. M. Azzazy, and M.-C. Wu, Biosens. Bioelectron., 1995, 10, 553.

24. A. K. Soe, S. Nahavandi, and K. Khoshmanesh, Biosens. Bioelectron., 2012, 35, 1.

25. Q. Liu, C. Wu, H. Cai, N. Hu, J. Zhou, and P. Wang, Chem. Rev., 2014, 114, 6423.

26. A. Savigner, P. Duchamp-Viret, X. Grosmaitre, M. Chaput, S. Garcia, M. Ma, and B. Palouzier-Paulignan, J. Neurophysiol., 2009, 101, 2898.
27. M. Reaz, M. Hussain, and F. Mohd-Yasin, Biol. Proced. Online, 2006, 8, 11.

28. S. Panigrahi, S. Sankaran, S. Mallik, B. Gaddam, and A. A. Hanson, Mater. Sci. Eng., C, 2012, 32, 1307.

29. H. J. Ko, J. H. Lim, E. H. Oh, and T. H. Park, "Bioelectronic Nose", 2014, Springer, 263.

30. S. Sankaran, L. R. Khot, and S. Panigrahi, Sens. Actuators, $B$, 2012, 171, 1.

31. R. Potyrailo and R. R. Naik, Ann. Rev. Mater. Res., 2013, 43, 307.

32. C. Ziegler, W. Göpel, H. Hämmerle, H. Hatt, G. Jung, L. Laxhuber, H.-L. Schmidt, S. Schütz, F. Vögtle, and A. Zell, Biosens. Bioelectron., 1998, 13, 539.

33. R. G. Smith, N. D'Souza, and S. Nicklin, Analyst, 2008, 133, 571.

34. S. Fanget, S. Hentz, P. Puget, J. Arcamone, M. Matheron, E. Colinet, P. Andreucci, L. Duraffourg, E. Myers, and M. Roukes, Sens. Actuators, B, 2011, 160, 804.

35. F. W. Sunderman, Ann. Clin. Lab. Sci., 2001, 31, 3.

36. E. J. Martinez-Finley, S. Chakraborty, S. J. Fretham, and M. Aschner, Metallomics, 2012, 4, 593.

37. X. Duan, E. Block, Z. Li, T. Connelly, J. Zhang, Z. Huang, X. Su, Y. Pan, L. Wu, and Q. Chi, Proc. Natl. Acad. Sci. U. S. A., 2012, 109, 3492.

38. V. Vodyanoy, BioMetals, 2010, 23, 1097. 